Artículo

\title{
Evaluación del comportamiento poscosecha de uchuva provenientes de sistemas de producción convencionales y agroecológicos
}

\author{
Aura Jessica Guevara Collazos ${ }^{1}$ \\ Edwin Andrés Villagran Munar ${ }^{1 \S}$ \\ Fabian Andres Velasquez Ayala ${ }^{2}$ \\ Krystle Danitza González Velandia ${ }^{3}$
}

${ }^{1}$ Facultad de Ingeniería-Corporación Universitaria Minuto de Dios. Sede Cundinamarca. Zipaquirá, Av. 15 núm. 1-22, Sur Barrio l ${ }^{\text {ra }}$ Fragüita, Colombia. CP. 250251. (agueva14@ uniminuto.edu.co). ${ }^{2}$ Facultad de Ingeniería-Universidad Nacional de Colombia. Calle 45 Carrera 30, Ciudad universitaria, Bogotá, Colombia. CP. 110151. (fvelasquez@unal.edu.co). ${ }^{3}$ Facultad de Ingeniería-Corporación Universitaria Minuto de Dios. Calle 81B núm. 72B-70, Bogotá, Colombia. CP. 110151. (kgonzalez@uniminuto.edu.co).

${ }^{\S}$ Autor para correspondencia: evillagranm@uniminuto.edu.co.

\section{Resumen}

La producción de uchuva (Physalis peruviana L.) en Colombia tiene un alto interés socioeconómico por su potencial comercial y el amplio número de productores de pequeña escala que integran el sistema productivo. Las zonas de cultivo en Colombia están localizadas en los pisos térmicos fríos de los departamentos de Boyacá, Cundinamarca y Antioquía. Recientemente, con el fin de buscar la sostenibilidad en la producción agrícola, la producción agroecológica ha tenido un creciente aumento en las zonas donde se establece este tipo de cultivo. Por lo tanto, el objetivo de esta investigación fue evaluar y comparar los comportamientos postcosecha del fruto de uchuva, obtenido bajo dos sistemas de producción agrícola (convencionales y agroecológicos) establecidos bajo condiciones climáticas de la sabana de Bogotá. La metodología consistió en la medición y cuantificación de las principales variables fisicoquímicas: peso, diámetro ecuatorial, firmeza, $\mathrm{pH}$, sólidos solubles totales, acidez, respiración y pérdida de peso durante los distintos estados de madurez. Estos valores fueron comparados en referencia a lo establecido en la NTC 4580. Los resultados obtenidos para cada propiedad mostraron diferencias significativas entre cada sistema productivo y cada estado de madurez evaluado, encontrando una sensibilidad más elevada al deterioro postcosecha en los frutos obtenidos mediante producción agroecológica.

Palabras clave: Physalis peruviana L., acidez, firmeza, índice de madurez.

Recibido: julio de 2019

Aceptado: septiembre de 2019 


\section{Introducción}

Los frutos de uchuva (Physalis peruviana L.) pertenecen a la familia de las solanáceas, este tipo de fruto se califica botánicamente en el grupo de las bayas, su forma es esférica u ovoide y tienen un color particular naranja amarillento y otra característica es que se encuentra envuelta en un cáliz o capacho, esta fruta es originaria de los andes suramericanos (Almanza y Espinosa, 1995). Su composición nutricional muestra altos contenidos de antioxidantes, hierro, fósforo, fibra y proteínas (Fischer et al., 2011), que le brindan propiedades de alimento funcional y medicinal (Puente et al., 2011).

En la actualidad Colombia es el mayor exportador de fruta fresca de uchuva (SENA, 2014). En el territorio nacional existen aproximadamente 1023 hectáreas establecidas en cultivos de uchuva con una producción total de 15711 t. El área total está distribuida en los departamentos de Boyacá, Cundinamarca y Antioquia con porcentajes de participación en la producción nacional de 40.47, 22.88 y $22.7 \%$ respectivamente. Por otro lado, en los departamentos de Santander, Norte de Santander, Nariño, Cauca y Tolima los porcentajes de participación están por debajo del $5 \%$ en cada uno de ellos (Agronet, 2016).

El sistema productivo predominante en un alto porcentaje de estos cultivos es la producción agrícola convencional, aunque se debe resaltar que en los últimos años ha aumentado el área dedicada a la producción orgánica y agroecológica de este producto y de otros de importancia económica a nivel nacional (Sánchez, 2017).

El enfoque de producción agroecológica se enmarca en principios ecológicos para el diseño y manejo de agroecosistemas sostenibles (Gliessman, 2000). Estos sistemas se suelen caracterizar caracteriza por no utilizar productos de origen sintético, ni por depender de insumos externos, se promueve la fertilización orgánica y un manejo preventivo integral del cultivo, este tipo de estrategias fue impulsado por el auge de la producción más limpia y la demanda de mercados verdes.

El cultivo de uchuva está considerado como un sistema productivo de interés económico por su potencial de exportación, el ecotipo Colombia, es apetecido en los mercados internacionales debido a sus adecuadas características organolépticas (color, sabor y aroma), obtenidas a partir de las condiciones agro climatológicas predominantes de los pisos térmicos templados y fríos de los andes colombianos, factor que permitió la expansión del cultivo a las diversas zonas que presenta este tipo de condiciones (Ligarreto et al., 2005). Adicionalmente la producción de uchuva genera un alto impacto social ya que es sistema productivo de pequeños productores (Balaguera, 2015).

El fruto de uchuva pertenece al grupo de los frutos climatéricos (Ávila et al., 2006), adicionalmente se clasifica como un alimento altamente perecedero (Balaguera et al., 2016). Este tipo de productos tienen una vida útil reducida principalmente por un proceso de madurez acelerado asociado a altas tasas de respiración, alta producción y sensibilidad al etileno y altos contenido de agua, estos factores generan pérdidas en calidad y cantidad del producto, que a priori se convierten en un limitante productivo (Kader, 2002), afectando la sostenibilidad de los sistemas de producción agrícola en especial en los países en vía de desarrollo (DNP, 2016). 
En Colombia para el año 2010 de acuerdo con estadísticas presentadas por la FAO en 2011 se perdieron en la etapa de poscosecha un total de $1426932 \mathrm{t}$ de frutas y hortalizas, de esta cifra $81 \%$ correspondió a frutas específicamente.

Para poder determinar las operaciones de acondicionamiento e infraestructura adecuada a utilizar en el periodo poscosecha, con la finalidad de alargar la vida útil y mantener la calidad de los productos agrícolas, es necesario conocer los cambios físicos que ocurren en el fruto, cambios generados por el proceso de maduración y principalmente por factores fisiológicos y bioquímicos propios de cada tipo de fruto, para el caso de la uchuva cultivada en sistemas agrícolas convencionales, existen diversos estudios que caracterizan el comportamiento en postcosecha (Lanchero et al., 2007; Fischer et al., 2011; Balaguera, 2015; Pinzon et al., 2015).

Por otro lado, la información del comportamiento poscosecha en sistemas productivos agroecológicos es escasa, algunas investigaciones han encontrado diferencias significativas en la etapa de poscosecha asociado al sistema de producción. Castelo et al. (2014) reportaron mejores parámetros de calidad en los frutos orgánicos y rendimientos similares en todos los sistemas de producción para tomate (Solanum lycopersicum). Asimismo, Campuzano et al. (2010) encontraron que no hay diferencia significativa entre sistemas de producción orgánica y convencional para el cultivo de banano Cavendish (Musa acuminata AAA). Aunque los frutos obtenidos de forma orgánica exhibieron una mayor vida útil influenciada por una menor tasa de respiración.

La presente investigación tuvo como objetivo la evaluación y comparación de las propiedades fisicoquímicas y fisiológicas de los procesos de maduración del fruto de uchuva (Physalis peruviana L.) cultivadas en formas convencional y agroecológica. Con la finalidad de generar información base que pueda ser utilizada a futuro para diseñar estrategias de manejo para la cosecha y poscosecha de productos agroecológicos, ya que la producción de Uchuva bajo este sistema productivo es reciente y requiere de este tipo de estudios.

\section{Materiales y métodos}

\section{Sitios experimentales}

La obtención de las muestras se realizó en dos sistemas, un sistema de producción agrícola convencional (SC) ubicado en el municipio de Subachoque departamento de Cundinamarca ( ${ }^{\circ}$ 56' 59.12" latitud norte, $74^{\circ} 11^{\prime} 35.78^{\prime}$ ' longitud oeste y una altitud de $2710 \mathrm{~m}$ ), donde predomina el clima frío semihúmedo, con unas condiciones medias de temperatura de $13{ }^{\circ} \mathrm{C}$ y precipitaciones entre 600 y $1200 \mathrm{~mm}^{2}$ ño $^{-1}$ (Climate-Data, 2016). El sistema de producción agroecológica (SA) está ubicado en el municipio de Guasca departamento de Cundinamarca ( $4^{\circ} 53^{\prime} 22.33^{\prime}$ ' latitud norte, $73^{\circ} 53^{\prime} 16.35^{\prime}$ ' longitud oeste y una latitud de $2620 \mathrm{~m}$ ), predomina el clima frío y húmedo, con temperatura media $13{ }^{\circ} \mathrm{C}$ y precipitaciones entre 800 y $2000 \mathrm{~mm}^{2} \tilde{a n o}^{-1}$ (Ideam, 2010).

\section{Material vegetal}

Para el desarrollo experimental se utilizaron frutos de uchuva fresca (Physalis peruviana L.) provenientes de plantas ecotipo Colombia, se recolectaron para el sistema de producción SC y SA frutos en estados de madurez (Em) entre 0 y 6, según la escala de la Norma Técnica Colombiana 
NTC 4580, Instituto Colombiano de Normas Técnicas y Certificación ICONTEC (1999). Los frutos fueron transportados hasta el laboratorio de química y agroecología de la Universidad Minuto de Dios (Temperatura máx $19{ }^{\circ} \mathrm{C}$ y mín $11{ }^{\circ} \mathrm{C}$, HR: 88\%), donde los frutos fueron desinfectados por el método de inmersión en una solución de cloro a 50 ppm.

\section{Mediciones}

Los datos reportados en las mediciones corresponden a la media de seis frutos por cada Em, donde realizaron 3 réplicas para cada medición, excepto para la determinación de la pérdida de peso.

\section{Peso fresco y diámetro ecuatorial}

El peso fresco de los frutos en cada Em se realizó con una balanza analítica de precisión marca OHAUS modelo EX225D (0.0001 g). Las dimensiones del diámetro del fruto se realizaron con un calibrador marca Mitutoyo modelo HL128.

\section{Firmeza}

La resistencia a la deformación de los frutos fue medida con el uso de un penetrómetro de frutas GY-3 (rango $0.5-12 \mathrm{~kg} \mathrm{~cm}^{-2}$ y $1-24 \mathrm{~kg} \mathrm{~cm}^{-2}$ exactitud \pm 0.1 ).

\section{pH y Acidez total titulable (ATT)}

La extracción del jugo se realizó mediante maceración de los frutos. Para determinar el pH del jugo se empleó un potenciómetro Hanna Instruments modelo PH211. La ATT expresada como (\%) de ácido cítrico se calculó realizando titulación con $\mathrm{NaOH}$ y se calculó el (\%) de ácido mediante la ecuación 1 tomada de la NTC 4580 (ICONTEC, 1999).

Ácido cítrico $(\%)=\frac{\mathrm{V}_{\mathrm{a}} * \mathrm{~N}}{\mathrm{~V}_{\mathrm{b}}} * \mathrm{~K} * 100$

Donde: $\mathrm{V}_{\mathrm{a}}$ es volumen de $\mathrm{NaOH}$ consumido $(\mathrm{mL}) ; \mathrm{V}_{\mathrm{b}}$ es peso en gramos de muestra utilizada (6 $\mathrm{g}$ ); $\mathrm{K}$ es peso equivalente del ácido predominante del fruto (ácido cítrico $0.064 \mathrm{~g} \mathrm{meq}^{-1}$ ); y $\mathrm{N}$ es normalidad del $\mathrm{NaOH}\left(0.1 \mathrm{meq}^{-1}\right)$.

\section{Sólidos solubles totales (SST) e índice de madurez (Im)}

Los SST expresados como grados $\left(^{\circ}\right)$ Brix fueron determinados utilizando un refractómetro digital Hanna Instruments modelo HL96801. El Im es obtenido mediante la ecuación 2.

$$
\operatorname{Im}=\frac{\mathrm{SST}}{\mathrm{ATT}}
$$

\section{Respiración}

Para determinar la producción de $\mathrm{CO}_{2}$ de los frutos de uchuva en cada Em y cada sistema productivo se siguió el procedimiento descrito en el trabajo desarrollado por Trinchero et al. (1999). El cual plantea el uso de un sistema estático cerrado y un análisis por cromatografía. 


\section{Pérdida de peso}

Para determinar la pérdida de peso de los frutos de uchuva; a través, del tiempo a condiciones ambientales del laboratorio de poscosecha, las muestras fueron clasificadas por tipo de sistema productivo (SC, SA) y Em. Se realizó un seguimiento al cambio del peso durante 19 días. La pérdida de peso (PP en \%) por día es obtenida a partir de la ecuación 3.

$\mathrm{PP}=\frac{\mathrm{Pi}-\mathrm{Pd}}{\mathrm{Pi}} * 100$

Donde: PP es la pérdida de peso (\%), Pi es peso inicial, Pd es peso diario.

\section{Diseño experimental y análisis de datos}

Para la evaluación de los dos sistemas de producción (SC y SA) en siete estados de madurez (Em: 0-6) se implementó un diseño experimental completamente al azar con arreglo factorial de 2 x 7 para un total de 14 tratamientos. Los resultados de las variables cuantificadas fueron analizados $y$ comparados mediante Anova simple de una vía, una vez fueron verificados los supuestos fundamentales de normalidad y homocedasticidad. La comparación de las medias se realizó por el método mínimas diferencias significativas (LSD) de Fischer a un nivel de confianza de 95\% ( $p \leq$ 0.05). El Anova fue desarrollado con el paquete estadístico del software R (R Development Core Team, 2017).

\section{Resultados y discusión}

\section{Peso fresco y diámetro del fruto}

Estos parámetros físicos son relevantes para definir las operaciones de acondicionamiento adecuadas que se deben realizar en el periodo poscosecha. Se encontraron diferencias significativas $(p \leq 0.05)$, para cada Em y entre SC y SA (Cuadro 1). Los valores máximos medios de peso fresco obtenidos fueron de $6.27 \pm 1.21$ g y $4.8 \pm 0.48$ g, Para Em 6 y Em 4 en SA y SC respectivamente. Los valores mínimos de peso fresco fueron obtenidos en el Em0 en SA y SC exhibiendo un valor de $1.31 \pm 0.19 \mathrm{~g}$ y $2.83 \pm 0.3 \mathrm{~g}$ respectivamente.

Cuadro 1. Valores medios de las variables peso (g) y diámetro ecuatorial $(\mathrm{cm})$ determinadas en frutos de uchuva (Physalis peruviana L.) Ecotipo Colombia.

\begin{tabular}{ccccc}
\hline $\begin{array}{c}\text { Sistema } \\
\text { productivo }\end{array}$ & Estado de madurez & Peso $(\mathrm{g})$ & Diámetro ecuatorial $(\mathrm{cm})$ & Firmeza $\left(\mathrm{kg} \mathrm{cm}^{-2}\right)$ \\
\hline SA & Em 0 & $1.31 \pm 0.19 \mathrm{a}$ & $1.34 \pm 0.09 \mathrm{a}$ & $5.35 \pm 0.76 \mathrm{a}$ \\
$\mathrm{SA}$ & Em 1 & $2.68 \pm 0.3 \mathrm{bcd}$ & $1.61 \pm 0.08 \mathrm{~b}$ & $4.05 \pm 0.72 \mathrm{bc}$ \\
$\mathrm{SA}$ & Em 2 & $2.98 \pm 0.21 \mathrm{~b}$ & $1.65 \pm 0.07 \mathrm{bc}$ & $4.46 \pm 0.57 \mathrm{~b}$ \\
$\mathrm{SA}$ & Em 3 & $3.86 \pm 0.48 \mathrm{efg}$ & $1.78 \pm 0.08 \mathrm{de}$ & $3.37 \pm 0.3 \mathrm{de}$ \\
$\mathrm{SA}$ & Em 4 & $3.19 \pm 0.32 \mathrm{bcde}$ & $1.64 \pm 0.07 \mathrm{~b}$ & $3.21 \pm 0.41 \mathrm{de}$ \\
$\mathrm{SA}$ & Em 5 & $4.23 \pm 0.67 \mathrm{gh}$ & $1.80 \pm 0.09 \mathrm{e}$ & $2.47 \pm 0.47 \mathrm{fg}$ \\
$\mathrm{SA}$ & Em 6 & $6.27 \pm 1.21 \mathrm{def}$ & $2.04 \pm 0,14 \mathrm{~g}$ & $2.25 \pm 0.26 \mathrm{~g}$ \\
\hline
\end{tabular}




\begin{tabular}{ccccc}
\hline $\begin{array}{c}\text { Sistema } \\
\text { productivo }\end{array}$ & Estado de madurez & Peso $(\mathrm{g})$ & Diámetro ecuatorial $(\mathrm{cm})$ & Firmeza $\left(\mathrm{kg} \mathrm{cm}^{-2}\right)$ \\
\hline $\mathrm{SC}$ & Em 0 & $2.57 \pm 0.47 \mathrm{~b}$ & $1.67 \pm 0.06 \mathrm{bcd}$ & $4.53 \pm 0.46 \mathrm{~b}$ \\
$\mathrm{SC}$ & Em 1 & $2.83 \pm 0.3 \mathrm{bcd}$ & $1.68 \pm 0.11 \mathrm{bcd}$ & $4.48 \pm 0.22 \mathrm{~b}$ \\
$\mathrm{SC}$ & Em 2 & $3.93 \pm 0.83 \mathrm{fg}$ & $1.86 \pm 0.14 \mathrm{ef}$ & $3.70 \pm 0.37 \mathrm{~cd}$ \\
$\mathrm{SC}$ & Em 3 & $3.32 \pm 0.62 \mathrm{cdef}$ & $1.76 \pm 0.1 \mathrm{cde}$ & $3.28 \pm 0.27 \mathrm{de}$ \\
$\mathrm{SC}$ & Em 4 & $4.8 \pm 0.48 \mathrm{~h}$ & $1.96 \pm 0.09 \mathrm{fg}$ & $2.91 \pm 0.46 \mathrm{ef}$ \\
$\mathrm{SC}$ & Em 5 & $3.78 \pm 0.66 \mathrm{efg}$ & $1.78 \pm 0.12 \mathrm{de}$ & $2.88 \pm 0.44 \mathrm{ef}$ \\
$\mathrm{SC}$ & Em 6 & $3.37 \pm 0.52 \mathrm{i}$ & $1.67 \pm 0.09 \mathrm{bcd}$ & $2.16 \pm 0.23 \mathrm{~g}$ \\
\hline
\end{tabular}

$\mathrm{SC}=$ Sistema de producción convencional. $\mathrm{SA}=$ sistema de producción agroecológica. $\mathrm{Em}=$ estado de madurez. Medias con letras distintas en cada de columna presentan diferencias significativas $(p \leq 0.05)$.

Los valores del diámetro ecuatorial de los frutos muestran diferencias significativas $(p<0.05)$, para cada Em y entre sistemas productivos (¡Error! No se encuentra el origen de la referencia.). La tendencia de los datos mostrada por este parámetro es similar a la obtenida para el peso fresco. Valores máximos del diámetro oscilaron entre $2.04 \pm 0.14 \mathrm{~cm}$ y $1.96 \pm 0.09 \mathrm{~cm}$ para los Em 6 y Em 4 en SA y SC respectivamente, mientras que los valores mínimos fueron de $1.34 \pm 0.09 \mathrm{~cm}$ y 1.67 $\pm 0.06 \mathrm{~cm}$ para los Em0 en SA y SC. Las diferencias encontradas para el peso fresco y el diámetro pueden estar fuertemente influencias por las diferentes prácticas culturales y agronómicas que se utilizan para cada sistema productivo, tales como riego, podas de formación y planes de fertilización (Martínez et al., 2008).

De acuerdo con estos resultados se concluye que los frutos de SC y SA se encuentran en un alto porcentaje dentro de los calibres B y C referenciados en la NTC 4580 (ICONTEC, 1999), a excepción del Em 0 de SA que se clasifica calibre A, aunque esto no es factor limitante para su comercialización, ya que la norma establece que se pueden tener frutos de las diferentes categorías sin importar su calibre.

\section{Firmeza}

El valor de la deformación generada a partir de una fuerza aplicada sobre un fruto suele asociarse con cambios estructurales a nivel celular y algunos cambios bioquímicos que ocurren en el pericarpio durante el proceso de maduración (Chitarra et al., 2005). Además, la pérdida de firmeza es un parámetro de calidad en frutos de uchuva en su etapa poscosecha. Esta variable mostró diferencias estadísticamente significativas $(p \leq 0.05)$, en un amplio porcentaje de los Em evaluados y los sistemas productivos SC y SA (Cuadro 1). En el Em0 para SA y SC se encontraron los valores medios máximos de firmeza $\left(5.35 \pm 0.76 \mathrm{~kg} \mathrm{~cm}^{-2}\right.$ y $4.53 \pm 0.46 \mathrm{~kg} \mathrm{~cm}^{-2}$, respectivamente).

No obstante, esta variable en función del tiempo muestra un comportamiento decreciente para los Em restantes hasta alcanzar valores mínimos de $2.25 \pm 0.26$ y $2.16 \pm 0.23 \mathrm{kgcm}^{-2}$ para el Em 6 en SA y SC respectivamente.

La pérdida de firmeza es un indicativo que durante el proceso de maduración se genera un deterioro de los frutos de uchuva, este estudio reveló que esta pérdida es gradualmente más acentuada en $\mathrm{SA}$, esto puede estar influenciado por las prácticas de fertilización usadas bajo este sistema 
producción, especialmente el manejo de elementos como calcio (Ca) y potasio (K) (Sanders, 2002). La pérdida de firmeza en uchuva está asociada al hidrólisis de polisacáridos estructurales y de reserva debido a la actividad de enzimas como la pectinmetilesterasa, la poligalacturonasa y unas glicosiladas (Trinchero et al., 1999), estas enzimas generan una degradación de la pared celular que provocan la pérdida de resistencia del fruto (Morais et al., 2008).

También se ha relacionado la presencia de la hormona etileno y su nivel de concentración con la pérdida de firmeza en uchuva (Balaguera et al., 2011).

\section{pH}

El cuadro 2 muestra el cambio del pH en función del estado de madurez por sistema productivo. En general, se presentaron diferencias significativas $(p \leq 0.05)$, entre los Em y los sistemas productivos. Valores más bajos de $\mathrm{pH}$ se presentan en SA $(3.85 \pm 0.07$ y $3.48 \pm 0.01)$. No obstante, existe un comportamiento similar entre SA y SC respecto a la disminución del valor del pH en los primeros estados de madurez y un posterior aumento de este. Estos cambios pueden estar asociados por al aumento de los ácidos orgánicos (Marschner, 1995).

El pH toma relevancia en frutas principalmente por ser un regulador del metabolismo, en la mayoría de frutas aproximadamente $90 \%$ del volumen celular es ocupado por un órgano celular denominado vacuola, que presenta pH por debajo de 5 ( Nanos et al., 1993; Novoa et al., 2006), resultados que soportan y dan validez a los obtenidos en esta investigación.

Cuadro 2. Valores medios de las variables pH, SST ( ${ }^{\circ}$ Brix), ATT (\% ácido cítrico) y Im, determinadas en frutos de uchuva (Physalis peruviana L.) Ecotipo Colombia.

\begin{tabular}{cccccc}
\hline $\begin{array}{c}\text { Sistema } \\
\text { productivo }\end{array}$ & $\begin{array}{c}\text { Estado de } \\
\text { madurez }\end{array}$ & $\mathrm{pH}$ & SST $\left({ }^{\circ}\right.$ Brix $)$ & ATT $(\%)$ ácido cítrico & Im (SST/ATT) \\
\hline $\mathrm{SA}$ & Em 0 & $3.85 \pm 0.07 \mathrm{a}$ & $6.46 \pm 0.23 \mathrm{a}$ & $2.62 \pm 0,03 \mathrm{a}$ & $2.46 \pm 0.05 \mathrm{a}$ \\
$\mathrm{SA}$ & Em 1 & $3.54 \pm 0.04 \mathrm{f}$ & $7.1 \pm 0.27 \mathrm{~b}$ & $2.45 \pm 0.05 \mathrm{c}$ & $2.89 \pm 0.11 \mathrm{~b}$ \\
$\mathrm{SA}$ & Em 2 & $3.49 \pm 0.01 \mathrm{fg}$ & $9.58 \pm 0.18 \mathrm{~d}$ & $2.29 \pm 0.02 \mathrm{e}$ & $4.18 \pm 0.03 \mathrm{~d}$ \\
$\mathrm{SA}$ & Em 3 & $3.48 \pm 0.01 \mathrm{~g}$ & $118 \pm 0.33 \mathrm{f}$ & $2.07 \pm 0.01 \mathrm{~g}$ & $5.70 \pm 0.09 \mathrm{f}$ \\
$\mathrm{SA}$ & Em 4 & $3.66 \pm 0.03 \mathrm{e}$ & $12.75 \pm 0.29 \mathrm{~h}$ & $1.91 \pm 0.02 \mathrm{i}$ & $6.65 \pm 0.09 \mathrm{~h}$ \\
$\mathrm{SA}$ & Em 5 & $3.69 \pm 0.05 \mathrm{de}$ & $13.41 \pm 0.22 \mathrm{ij}$ & $1.78 \pm 0.01 \mathrm{j}$ & $7.47 \pm 0.15 \mathrm{i}$ \\
$\mathrm{SA}$ & Em 6 & $3.64 \pm 0.04 \mathrm{e}$ & $14.53 \pm 0.21$ & $1.66 \pm 0.08 \mathrm{k}$ & $8.74 \pm 0.02 \mathrm{j}$ \\
$\mathrm{SC}$ & Em 0 & $3.77 \pm 0.1 \mathrm{bc}$ & $8.03 \pm 0.2 \mathrm{c}$ & $2.51 \pm 0.05 \mathrm{~b}$ & $3.20 \pm 0.13 \mathrm{c}$ \\
$\mathrm{SC}$ & Em 1 & $3.73+0.08 \mathrm{~cd}$ & $9.48 \pm 0.38 \mathrm{~d}$ & $2.36 \pm 0.03 \mathrm{~d}$ & $4.01 \pm 0.11 \mathrm{~d}$ \\
$\mathrm{SC}$ & Em 2 & $3.69 \pm 0.05 \mathrm{de}$ & $11.43 \pm 0.18 \mathrm{e}$ & $2.25 \pm 0.02 \mathrm{f}$ & $5.07 \pm 0.02 \mathrm{e}$ \\
$\mathrm{SC}$ & Em 3 & $3.75 \pm 0.06 \mathrm{~cd}$ & $12.36 \pm 0.15 \mathrm{~g}$ & $2.02 \pm 0.03 \mathrm{~h}$ & $6.11 \pm 0.05 \mathrm{~g}$ \\
$\mathrm{SC}$ & Em 4 & $3.69 \pm 0.01 \mathrm{de}$ & $13.21 \pm 0.08 \mathrm{i}$ & $1.78 \pm 0.05 \mathrm{j}$ & $7.43 \pm 0.07 \mathrm{i}$ \\
$\mathrm{SC}$ & Em 5 & $3.74 \pm 0.01 \mathrm{~cd}$ & $13.58 \pm 0.17 \mathrm{j}$ & $1.52 \pm 0.091$ & $8.95 \pm 0.03 \mathrm{k}$ \\
$\mathrm{SC}$ & Em 6 & $3.82 \pm 0.05 \mathrm{ab}$ & $14.18 \pm 0.21 \mathrm{k}$ & $1.24 \pm 0.13 \mathrm{~m}$ & $11.44 \pm 0.131$ \\
\hline
\end{tabular}

$\mathrm{SC}=$ sistema de producción convencional. $\mathrm{SA}=$ sistema de producción agroecológica. Em= estado de madurez. Medias con letras distintas en cada de columna presentan diferencias significativas $(p \leq 0.05)$. 


\section{Sólidos solubles totales (SST)}

En el jugo del fruto de uchuva el porcentaje de azúcares presente en el contenido de SST se encuentra en rangos de 80 a 95\% (Fischer y Martínez, 1999). Los SST exhibieron un comportamiento que presentó diferencias estadísticamente significativas $(p \leq 0.05)$ para cada Em y para cada sistema productivo. El comportamiento de los SST presenta una tendencia creciente durante el proceso de maduración, lo cual está asociado a los procesos de hidrolisis de la pared celular, donde los polisacáridos son degradados a monosacáridos (Menendez et al., 2006).

Para este estudio se obtuvieron unos valores mínimos de $6.46 \pm 0.23$ y $8.03 \pm 0.2{ }^{\circ}$ Brix, para el Em en SA y SC respectivamente, los valores máximos se obtuvieron en el Em 6 con $14.18 \pm 0.21$ para $\mathrm{SA}$ y $14.53 \pm 0.2$ en $\mathrm{SC}$, se debe mencionar que ninguno de los Em cumplió con el requerimiento mínimo de ${ }^{\circ}$ Brix recomendado por la NTC 4580. Lo anterior, puede estar correlacionado con la localización de los sistemas productivos. Dado que estos se encuentran por encima de los 2300 msnm, estudios realizados por (Fischer et al., 2007) han demostrado que estos niveles de altura generan una reducción de los SST y la concentración de sacarosa.

\section{Acidez total titulable (ATT)}

El valor de ATT está relacionado directamente con la presencia de ácidos en el fruto de uchuva, los ácidos característicos para este fruto son el cítrico, tartárico, málico y el oxálico (Pinzon et al., 2015). El comportamiento observado en SA y SC mostró una disminución del porcentaje de acidez para cada Em (Cuadro 2). Este es un comportamiento típico durante el proceso de maduración del fruto de uchuva (Balaguera et al., 2011). La disminución de la ATT está asociada al uso de los ácidos orgánicos como sustratos de respiración (Kader, 2002) o su transformación mediante glucogénesis a azúcares.

Los valores obtenidos exhibieron diferencias significativas $(p \leq 0.05)$ entre Em y los sistemas productivos evaluados, el valor máximo medio obtenido fue de $2.62 \pm 0.23$ y $2.51 \pm 0.23 \%$ para el Em 0 en SA y SC respectivamente, el valor mínimo medio fue de $1.66 \pm 0.23$ y $1.24 \pm 0.23 \%$, para el Em6 en SA y SC (Cuadro 2), los valores obtenidos en los Em 0-6 para los dos sistemas productivos estuvieron por debajo del valor recomendado por la NTC 4580 (ICONTEC, 1999).

\section{Índice de madurez (SST/ATT)}

Este valor es un indicativo del sabor del fruto y de su estado de madurez (Balaguera, 2015) por lo tanto es un parámetro de aceptación comercial. Se observó un comportamiento creciente, para los dos sistemas productivos, comportamiento asociado al aumento generalizado de los SST para cada Em y la reducción de la acidez para estos mismos estados (Cuadro 2). Se encontraron diferencias significativas $(p \leq 0.05)$ para SA y SC, y de igual manera entre los Em.

Para el SA se puede mencionar que los valores de índices de madurez para la totalidad de los Em, estuvo por debajo del valor mínimo recomendado en la NTC 4580; esto quiere decir, que estos frutos en términos de calidad organoléptica para el componente del sabor presentan desventajas en comparación con los producidos en SC, puesto que los valores obtenidos de índice de madurez para los Em 3-6 en SC están por encima del mínimo recomendado. 


\section{Patrón respiratorio}

Los frutos de uchuva son considerados como climatéricos, porque aumentan su patrón respiratorio después de obtener su madurez fisiológica (Balaguera, 2015). Los valores obtenidos para SA y SC presentaron una tendencia similar bajo las condiciones ambientales presentes en el laboratorio (Figura 1). Se encontraron diferencias significativas entre Em y los sistemas de producción, para SA se presenta un patrón respiratorio que presenta un valor máximo inicial por unidad de peso de $7.05 \pm 0.33 \mathrm{mg} \mathrm{CO}_{2} \mathrm{~g}^{-1} \mathrm{~d}^{-1}$ para el Em 1, luego decae hasta un valor de $2.89 \pm 0.03 \mathrm{mg} \mathrm{CO}_{2} \mathrm{~g}^{-1} \mathrm{~d}^{-1}$ en el Em 5, para posteriormente aumentar hasta un valor de $7.3 \pm 0.1 \mathrm{mg} \mathrm{CO}_{2} \mathrm{~g}^{-1} \mathrm{~d}^{-1}{ }_{(\mathbf{i}}$ Error! No se encuentra el origen de la referencia.).

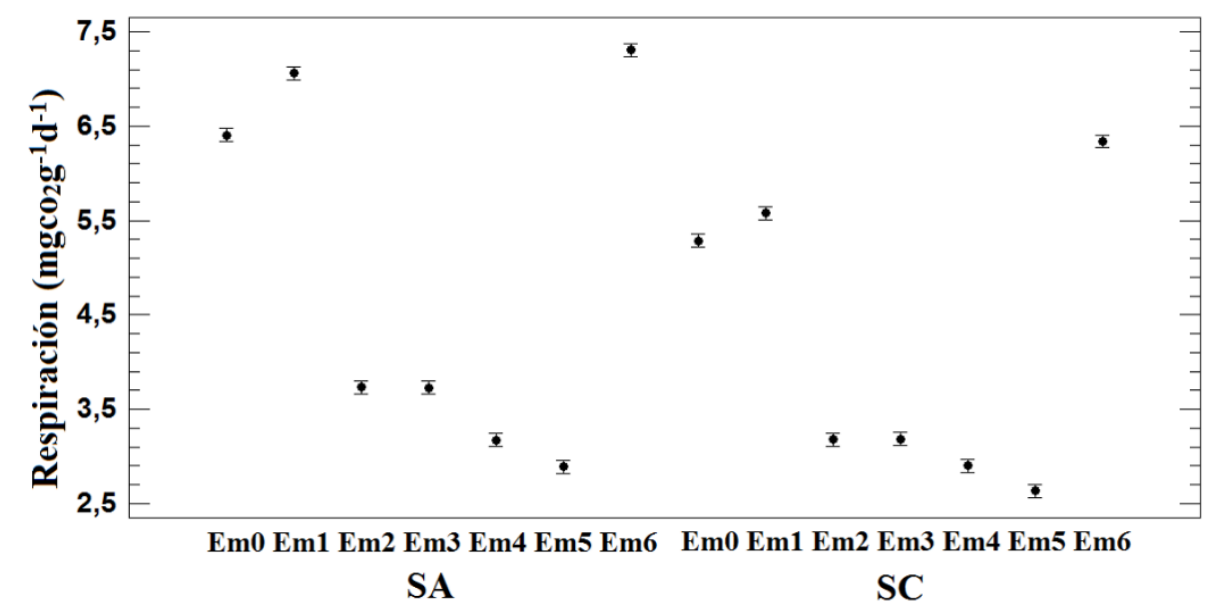

Figura 1. Comportamiento de las tasas de respiración para ca Em en SA y SC.

Para el SC se obtuvo un máximo inicial de $5.58 \pm 0.08 \mathrm{mg} \mathrm{CO}_{2} \mathrm{~g}^{-1} \mathrm{~d}^{-1}$, este valor disminuyó hasta $2.9 \pm 0.04 \mathrm{mg} \mathrm{CO}_{2} \mathrm{~g}^{-1} \mathrm{~d}^{-1}$ en el Em 4 y luego aumentó hasta $6.33 \pm 0.03 \mathrm{mgCO}_{2} \mathrm{~g}^{-1} \mathrm{~d}^{-1}$ en el Em 6 . Este comportamiento es explicado principalmente porque la uchuva presenta una característica propia a este tipo de fruto y es el aumento de tamaño y peso mientras se encuentra ligada a la planta durante su fase de maduración, algo previamente demostrado por Trinchero et al. (1999), estos resultados permiten concluir que la tasa de respiración de uchuva producida en SA es más elevada, por lo tanto su proceso de deterioro es más acelerado lo que se traduce en una vida útil en poscosecha más reducida en comparación con SC.

\section{Pérdida de peso}

La pérdida de peso en productos frutícolas perecederos presenta una tendencia creciente en el tiempo, esta pérdida se asocia al proceso de maduración y más directamente a los procesos de respiración y transpiración del fruto, adicionalmente puede ser acelerada por factores como, daño mecánico o el ataque de patógenos. La Figura 2, presenta el comportamiento porcentual de pérdida de peso para SC y SA, se encontró que para el día 5 el valor de pérdida para SA fue de 21.08, 10.31, $11.09,12.04,10.51,16.72$ y $8.02 \%$ para los Em del 0 al 6 respectivamente, mientras que para el SC fueron de 14.73, 9.93, 6.87, 5.59, 6.54 y 9.78 . 
Para los Em del 0 al 6, valores que se encuentran por debajo de los obtenidos en SA, ésta tendencia se mantuvo para el día 10 y se reafirmó en el día 15 donde se obtuvieron valores para SA de 42.08, $38.02,56.93,49.11,58.74,89.12$ y 43.54 para los Em del 0 al 6, mientras que para el SC se obtuvieron valores de 33.46, 24.88, 22.19, 17.57, 43.44, 35.09 y 56.46\% para los Em del 0 al 6. Para los días posteriores se puede observar cómo los Em 0-3 del SC son los que menos pérdida porcentual de peso acumulan (Figura 2a).
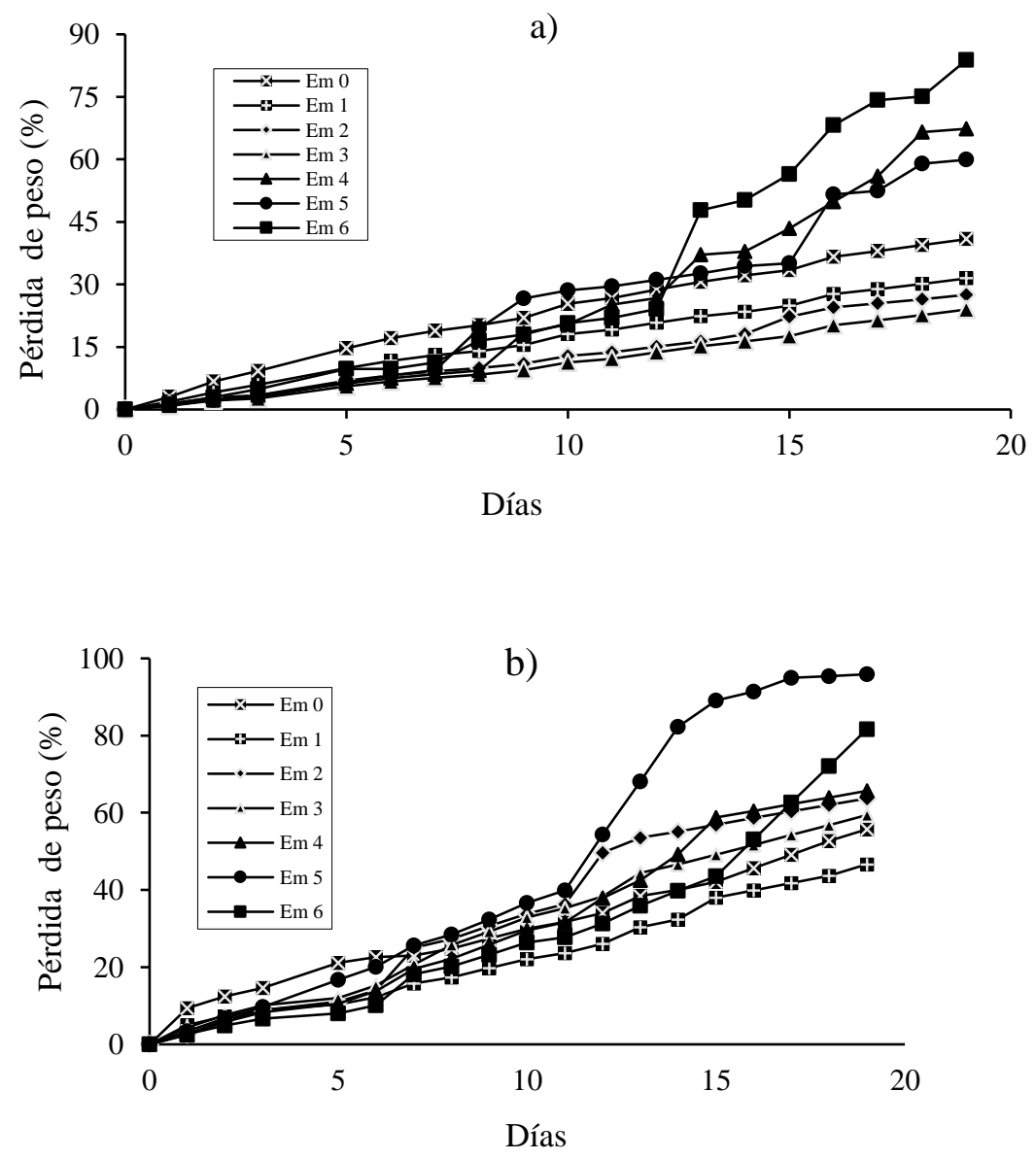

Figura 2. Pérdida de peso (\%) en frutos de uchuva (Physalis peruviana L.) Ecotipo Colombia, a) sistema de producción agrícola convencional (SC); y b) sistema de producción agroecológica (SA).

\section{Conclusiones}

Para los sistemas de producción SA y SC, las características fisicoquímicas como, SST, ATT, firmeza y $\mathrm{pH}$, presentaron diferencias significativas en un alto porcentaje de los Em, debido principalmente a los procesos fisiológicos y bioquímicos que tienen origen en el proceso de maduración de frutos climatéricos. En términos generales se observó cómo los frutos producidos en SA presentan una mayor tasa de respiración, una pérdida de firmeza más acentuada durante el proceso de madurez, una tasa porcentual de pérdida de peso más elevada, lo que conlleva a una vida útil en poscosecha más restringida en comparación con los frutos obtenidos en SC, lo 
que demuestra que la vida útil de una uchuva obtenida bajo un sistema de producción convencional es mayor.

Es recomendable que estudios futuros, evalúen un mayor número de fincas bajo estos mismos sistemas productivos en diversas condiciones climáticas, identificando las características mínimas de la uchuva obtenida bajo producción agroecológica y su comportamiento bajo diferentes escenarios de almacenamiento que permitan comparar las variables obtenidas con establecidas por la NTC 4580, ya que los frutos obtenidos en SA de esta investigación no alcanzaron los niveles mínimos de índice de madurez reportados en la norma, este índice de madurez relaciona el componente de sabor del fruto y es un factor que puede ser castigado comercialmente, a su vez es un parámetro que no depende de la etapa de poscosecha directamente, si no de factores pre cosecha que pueden ser climáticos, agronómicos o fisiológicos.

\section{Agradecimientos}

Los autores agradecen a la Corporación Universitaria Minuto De Dios (UNIMINUTO) y a los productores de las fincas La Tata (Subachoque) y San Luis (Guasca) Conflicto de intereses: el manuscrito fue preparado y revisado con la participación de todos los autores, quienes declaramos que no existe conflicto. Financiación: recursos propios e internos de la universidad.

\section{Literatura citada}

Agronet. 2016. Producción nacional por producto, uchuva. http://www.agronet.gov.co.

Almanza, P. J. y Espinosa, C. J. 1995. Desarrollo morfológico y análisis fisicoquímico de frutos de uchuva (Physalis peruviana L.) para identificar el momento óptimo de cosecha. Trabajo final. Especialización en frutales de clima frío, Facultad de Ciencias Agropecuarias, Universidad Pedagógica y Tecnológica de Colombia (UPTC), Tunja. 83 p.

Ávila, J. A.; Moreno, P. y Fischer, G. 2006. Influencia de la madurez del fruto y del secado del cáliz en uchuva (Physalis peruviana L.), almacenada a $18{ }^{\circ} \mathrm{C}$. Acta Agron. (Colombia). 55(4):29-37.

Balaguera, L. H. E.; Martínez, C. y Herrera, A. A. 2011. Papel del cáliz en el comportamiento poscosecha de frutos de uchuva (Physalis peruviana L.) ecotipo Colombia. Rev. Colomb. Cienc. Hortíc. 8(2):181-191.

Balaguera, L. H. E.; Martínez, C. y Herrera, A. A. 2016. Comportamiento poscosecha de frutos de Uchuva (Physalis peruviana L.): efecto de diferentes dosis y tiempos de exposición al 1Metilciclopropeno. Bioagro. 28(1):021-028.

Balaguera, H. E. 2015. Comportamiento poscosecha del fruto de uchuva (P. peruviana L.): efecto del 1-metilciclopropeno y de la refrigeración. Tesis de Doctorado en Ciencias agrarias. Facultad de Ciencias Agrarias, Universidad Nacional de Colombia, Bogota. 251 p.

Campuzano, A.; Cornejo, F.; Rui, O. y Peralta, E. 2010. Efecto del tipo de producción de banano cavendish en su comportamiento poscosecha. Revista Tecnológica ESPOL. 23(2):41-48.

Castelo, G. A.; Gutiérrez, C. M.; Arellano, G. M.; Castro, E. L.; Lares, V. F.; Figueroa, L. P. 2014. Calidad postcosecha y de rendimiento de tomate $c v$ grandella bajo un sistema de producción orgánica y convencional en condiciones de casa sombra. In: XXXIX Congreso Nacional de la Ciencia del Suelo, Ciudad Juárez, Chihuahua, 19 al 24 octubre 2014. 
Chitarra, M. I. F. e Chitarra, A. B. 2005. Pos-colheita de frutos e hortalicas: fisiologia e manuseio. Lavras: ESAL-FAEPE. 785 p.

Climate-data. 2016. Climate-data. http://es.climate-data.org/.

DNP. 2016. Departamento Nacional de Planeación. Pérdida y desperdicio de alimentos en Colombia. Estudio de la Dirección de Seguimiento y Evaluación de Políticas Públicas. 43 p.

FAO. 2011. Global food losses and food waste. Extent, causes and prevention. http://www.fao.org/docrep/014/mb060e/mb060e00.htm

Fischer, G.; Ebert, G. and Lüdders, P. 2007. Production, seeds and carbohydrate contents of cape gooseberry (Physalis peruviana L.) fruits grown at two contrasting Colombian altitudes. J. Appl. Bot. Food Quality. 81(1):29-35.

Fischer, G.; Herrera, A. and Almanza, P. J. 2011. 17-Cape gooseberry (Physalis peruviana L.). In: postharvest biology and technology of tropical and subtropical fruits. 374-397e p. https://doi.org/10.1533/9780857092762.374.

Fischer, G. y Martínez, O. 1999. Calidad y madurez de la uchuva (Physalis peruviana L.) en relación con la coloración del fruto. Agronomía Colombiana. 16(1-3):35-39.

Gliessman, S. R. 2000. Agroecosystem sustainability: developing practical strategies. Boca Raton, FL: CRC/Taylor and Francis. 224 p.

ICONTEC. 1999. Instituto colombiano de normas técnicas y certificación. Frutas frescas. Uchuva. Especificaciones. Norma Técnica Colombiana NTC 4580.

IDEAM. 2010. Instituto de Hidrología, Meteorología y Estudios Ambientales. Estudio Nacional del Agua. Bogotá. 409 p.

Kader, A. A. 2002. Postharvest technology of horticultural crops. $3^{\text {th }}$ (Ed.). Division of Agriculture and Natural Resources. ANR Publications, University of California, Oakland, CA. 535 p.

Lanchero, O.; Velandia, G.; Fischer, G. y Varela, N. C. 2007. Comportamiento de la uchuva (Physalis peruviana L.) en poscosecha bajo condiciones de atmósfera modificada activa. Rev. Corpoica - Ciencia y Tecnología Agropecuaria. 8(1):61-68.

Ligarreto, G.; Lobo, M.; Correa, Y. A. 2005. Recursos genéticos del género Physalis en Colombia. In: Fischer, G.; Miranda, D.; Piedrahita, W. y Romero, J. (Ed.). Avances en cultivo, poscosecha y exportación de la uchuva (Physalis peruviana L.) en Colombia, Unibiblos, Universidad Nacional de Colombia. Bogotá. 9-27 pp.

Marschner, H. 1995. Mineral Nutrition of Higher Plants. Second E. Marschner (Ed.). San Diego: Academic Press. 595 p.

Martínez, F. E.; Sarmiento, J.; Fischer, G.; Jiménez, F. 2008. Efecto de la deficiencia de N, P, K, $\mathrm{Ca}, \mathrm{Mg}$ y B en componentes de producción y calidad de la uchuva (Physalis peruviana L.). Agronomía Colombiana. 26(3):389-398.

Menéndez, O.; Lozano, S. E.; Arenas, M.; Bermúdez, K.; Martínez, A. y Jiménez, A. 2006. Cambios en la actividad de $\alpha$-amilasa, pectinmetilesterasa y poligalacturonasa durante la maduración del maracuyá amarillo (Passiflora edulis var. flavicarpa Degener). Interciencia. 31(10):728-733.

Morais, P. L. D.; Miranda, M. R. A.; Lima, L. C. O.; Alves, J. D.; Alves, R. E. and Silva, J. D. 2008. Cell wall biochemistry of sapodilla (Manilkara zapota) submitted to 1methylcyclopropene. Braz. J. Plant Physiol. 20(2):85-94.

Nanos, G. D. Kader, Y. A. A. (1993). Low $\mathrm{O}_{2}$ induced changes in $\mathrm{pH}$ en energy change in pear fruit tissue. Postharv. Biol. Technol. 3(3):285-291.

Novoa, R.; Bojacá, M.; Galvis, J. y Fischer, G. 2006. La madurez del fruto y el secado del cáliz influyen en el comportamiento poscosecha de la uchuva, almacenada a $12 \mathrm{C}$ (Physalis peruviana L.). Agron. Colomb. 24(1):77-86. 
Pinzon. H.; Reyes. J.; Alvarez. J.; Leguizamo. M.F. y Joya, G. 2015. Comportamiento del fruto de uchuva Physalis peruviana L., bajo diferentes temperaturas de almacenamiento. Rev. Cienc. Agr. 32(322):26-3526.

Puente, L. A.; Pinto, M. C. A.; Castro, E. S. and Cortés, M. 2011. Physalis peruviana Linnaeus, the multiple properties of a highly functional fruit: A review. Food Research International. 44(7):1733-1740. https://doi.org/10.1016/j.foodres.2010.09.034.

R Development Core Team. 2017. R: A language and environment for statistical computing. Vienna: $\mathrm{R}$ Foundation for Statistical Computing. Recuperado de: http://www.Rproject.org.

Sánchez, C. J. 2017. Ecological agricultural products market in Colombia. Suma negocio. $8(18): 156-163$.

Sanders, D. 2002. Boron: it can work wonders. Grower guidelines, North Carolina State. 58 p.

SENA. 2014. Manual del cultivo de técnico Uchuva, buenas prácticas agrícolas. Medellín, Colombia. $111 \mathrm{p}$.

Trinchero, G. D.; Sozzi, G. O.; Cerri, A. M.; Vilella, F. and Fraschina, A. A. 1999. Ripening-related changes in ethylene production, respiration rate and cell-wall enzyme activity in goldenberry (Physalis peruviana L.), a solanaceous species. Postharvest Biol. Technol. 16(2):139-145. 